\title{
Hydrogen Bonding: A Bottom-Up Approach for the Synthesis of Films Composed of Gold Nanoparticles
}

\author{
Mariana Chirea, ${ }^{\mathrm{a} *}$ Carlos M Pereira, ${ }^{\mathrm{b} *}$ Fernando Silva \\ CIQ-UP L4, Departamento de Química da Faculdade de Ciências da Universidade do Porto, \\ Rua do Campo Alegre, 687, 4169-007 Porto (Portugal) \\ a mariana.chirea@fc.up.pt ; ${ }^{b^{*}}$ cmpereir@fc.up.pt \\ received paper15.01.2008 received revised paper 12.02.2008, accepted date, 15.02.2008
}

Keywords: gold nanoparticles, place exchange reaction, hydrogen bonding, self-assembling, solid films

Abstract. Citrate gold nanoparticles of $13.5 \mathrm{~nm}$ average diameters have been synthesized by Turkevich method and further functionalized by place exchange reaction using mercaptosuccinic acid (MSA), 1,4-benezenedimethanethiol (1,4BDMT) or mercaptoundecanoic acid (MUA) as the incoming ligands. In the first case, the functionalization of the gold clusters with MSA yielded solution of blue color and large aggregates on carbon grids. Moreover, after filtration and drying, any film formed by hydrogen bonding, could not be removed from the filter, being embedded into the filter texture. The second ligand used for the place exchange reaction, the 1,4BDMT, determined the change of the solution color from red to dark purple, and generation of a purple powder but no film formation. In the third case, the purification and drying of the MUA functionalized gold clusters yielded films of purple color with homogenous composition, robustness, soft topographies and remarkable optical properties which were dependent on their thicknesses. The Au-MUA NP films obtained are new, being generated by hydrogen bonding and having features which makes them very attractive for the fabrication of optoelectronic devices. The long alkyl chained thiol proved to be the adequate ligand for film synthesis. 


\section{Introduction}

The preparation of new materials with improved optical and electrical properties represents a continuous challenge in the nanotechnology research. Among all known inorganic nanoparticles, gold nanoparticles are the most studied nanomaterials due to their great chemical stability and their outstanding optical, electrical and catalytic properties.[1] The immobilization of nanoparticles in well-controlled 2D or 3D assemblies will facilitate their integration in future devices [2]. For this purpose various strategies have been used to achieve multidimensional nanoparticle assemblies: either the layer-by-layer self-assembly approach based on electrostatic, covalent, coordination or hydrogen interaction,[3-12] or the mediator-templating approach based on the interconnection of stabilized nanoparticles by bi- or multifunctional linkers assembling the nanoparticles through place-exchanging of the stabilizing ligands on their surface.[13-16] Among them, non-covalent interaction, such as hydrogen bonding, represents a simple method for the assembly of the nanoparticles without external manipulation, such as stirring which usually improves the electrostatic self-assembly of charged species in solution.[16,17-20] Hydrogen bonds are more dynamic and flexible in geometry than rigid covalent bonds being very important in the design of supramolecular structures.[21] Most methods to assemble inorganic nanoparticles by hydrogen bonding depend on biomolecular structures, in particular DNA-strands[22,23] and substrate/receptor pairs like biotin and streptavidin [24,25] or polymers dressed with hydrogenbonding moieties.[26] These approaches are characterized by the large size of the binding material compare to the dimensions of the particle cores and this limits their use in applications that depend on the particle-particle interaction. A simple method to overcome this problem is the functionalization of the nanoparticles with substantially smaller molecules. Their functionalization could be very easily achieved by place exchange reaction.[27] In this work we report the synthesis of large gold nanoparticles by Turkevich method, [28] and their further functionalization by place exchange reaction using three different incoming ligands: mercaptosuccinic acid, 1,4benzendimethanethiol or mercaptoundecanoic acid (see Scheme 1 representing the chemical structure of the gold nanoparticles obtained by place exchange reaction). Initially, our purpose was to study the size effect of MUA stabilized gold nanoparticles for the electrochemical sensing of various compounds in solution. For this reason, larger gold nanoparticles were synthesized by Turkevich method and successively functionalized with MUA, because the direct synthesis of gold clusters stabilized by MUA yields only clusters no larger than $4 \mathrm{~nm}$.[3] Interestingly, after purification, filtration and drying, these large MUA functionalized gold clusters generated, spontaneously, soft solid films based on hydrogen bonding interaction as it will be proved further in this work. This behavior has not been observed previously for small gold nanoparticles (1-4 nm) stabilized by the same ligand.[3] In order to test other types of film formation, two different ligands, the mercaptosuccinic acid and 1,4benezenedimethanethiol, were used for the functionalization of the citrate gold nanoparticles and the resulted clusters were characterized by UV-Vis spectroscopy and transmission electron microscopy. Although, an evident change of solution color was observed, no film formation was detected for the MSA and 1,4BDMT functionalized gold clusters. The new Au-MUA NP films were further characterized by UV-Vis spectroscopy, FT-IR Spectroscopy, Atomic Force Microscopy and Scanning Electron Microscopy. Interestingly, the Au MUA NP films showed robustness and optical properties dependent on their thicknesses, very soft topographies and homogenous composition. These features could make the films obtained very attractive for the fabrication of electronic devices, based on interdigitated arrays, due to the facility to cut them in various shapes or cast them into forms. Moreover, their optical properties can be tuned over a range of optical absorbances as a function of their thickness which allows the fabrication of optical sensors starting from the molecular level up to the micrometer level, depending on the amount of nanoparticles incorporated into the film. 
Place exchange reaction $(72 \mathrm{~h})$

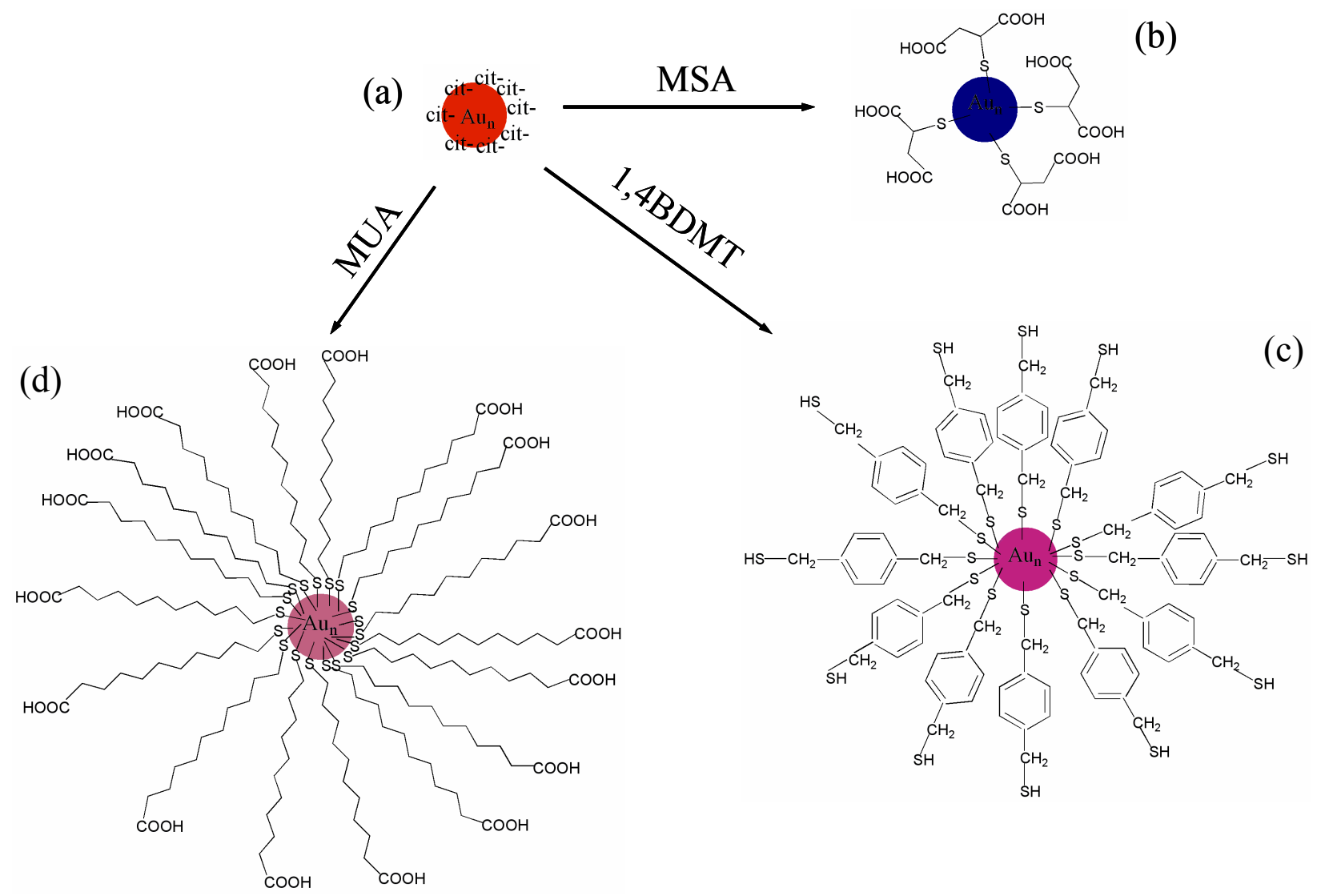

Scheme 1. Chemical structures of trisodium citrate gold nanoparticles (a) and the resulted functionalized gold clusters: mercaptosuccinic acid functionalized nanoparticles (b), 1,4 benzenedimethanethiol functionalized nanoparticles (c)and mercaptoundecanoic acid functionalized nanoparticles (d).

\section{Experimental section.}

Chemicals. Hydrogen tetrachloroaurate (III) trihydrate (99.9\%), mercaptosuccinic acid, (HOOC$\mathrm{CH} 2-\mathrm{CH}(\mathrm{SH}) \mathrm{COOH}, \quad 98 \%), 1,4$ benzenedimethanethiol ( $\mathrm{HS}_{-}-\mathrm{CH}_{2}-\mathrm{C}_{6} \mathrm{H}_{4}-\mathrm{CH}_{2}-\mathrm{SH}, \quad 98 \%$ ), mercaptoundecanoic acid $\left(\mathrm{HS}\left(\mathrm{CH}_{2}\right)_{10} \mathrm{COOH}, 98 \%\right.$,) and methanol $(99.8 \%)$ were purchased from Sigma Aldrich. Citrate trisodium dihydrate $\left(\mathrm{HOC}(\mathrm{COONa})\left(\mathrm{CH}_{2} \mathrm{COONa}\right)_{2} \cdot 2 \mathrm{H}_{2} \mathrm{O}\right.$ p.a.) was obtained from Merck. The glassware and magnetic stirring beads were cleaned prior to use with freshly prepared aqua regia $(\mathrm{HNO} / \mathrm{HCl} 1: 3, \% \mathrm{v})$ followed by abundantly rinsing with ultra-pure millipore water (resistivity $>18 \mathrm{M} \Omega \cdot \mathrm{cm}$ ) and dried.

\section{Synthesis of trisodium citrate stabilized gold nanoparticles:}

A $5 \times 10^{-6} \mathrm{~mol}_{\text {of }} \mathrm{HAuCl}_{4}$ was dissolved in $19 \mathrm{~mL}$ of deionized water and heated until boiling. Under continuing heating and strong stirring, $2 \mathrm{~mL}$ of sodium citrate of $0.5 \%$ aqueous solution was added. The color of the solution gradually changed from light yellow to faint purple, dark purple and finally settled on the well-known red-wine color of citrate stabilized gold nanoparticles (Figures 2a, 3a, 4a). 


\section{Trisodium citrate gold nanoparticles functionalization by place exchange reaction with MSA:}

After cooling at room temperature, the citrate gold nanoparticles solution was diluted with $20 \mathrm{~mL}$ of Millipore water and successively $6.8 \times 10^{-5}$ mol of mercaptosuccinic acid dissolved in $50 \mathrm{~mL}$ methanol was added under strong stirring. After 24 hours time of reaction, a second amount of thiol $\left(2 \times 10^{-3} \mathrm{~mol} \mathrm{MSA} / 50 \mathrm{~mL}\right.$ methanol) was added and stirred for additionally 36 hours. The final solution showed blue color and a second peak $(625 \mathrm{~nm})$ in the visible region of the spectra (See Figure 2b). Repeated centrifugation and sonication in water/methanol solution allowed the purification of the MSA functionalized gold clusters which were filtered on pre-cut membranes with pore diameters of $0.2 \mu \mathrm{m}$. The dry powder embedded within the filter texture showed blue color. No further recovery of the clusters was possible.

Trisodium citrate gold nanoparticles functionalization by place exchange reaction with 1,4 Benzenedimethanethiol:

Freshly prepared citrate gold nanoparticles of $13.5 \mathrm{~nm}$ average diameters were functionalized by mixing with $3.4 \times 10^{-5}$ mol of 1,4 Benzenedimethanethiol dissolved in $100 \mathrm{~mL}$ methanol, under strong stirring over 24 hours. A second amount of thiol was added $\left(6.5 \times 10^{-4} \mathrm{~mol} / 100 \mathrm{~mL}\right.$ methanol $)$ and stirred for additionally 36 hours. The resulted functionalized clusters were purified and filtered on membranes. The procedure yielded purple powders of 1,4 Benzenedimethanethiol functionalized gold clusters.

Trisodium citrate gold nanoparticles functionalization by place exchange reaction with mercaptoundecanoic acid:

The citrate gold nanoparticles solution freshly prepared, as described previously, was diluted with $20 \mathrm{~mL}$ of Millipore water, and successively $3.4 \times 10^{-5} \mathrm{~mol}$ of mercaptoundecanoic acid dissolved in $50 \mathrm{~mL}$ methanol were added under strong stirring. After 24 hours of continuous stirring at room temperature, a second amount of thiol was added $\left(6.8 \times 10^{-4}\right.$ mol dissolved in $100 \mathrm{~mL}$ methanol) in the solution mixture and stirred for 36 hours (Figure 3b). Next, the solvent was removed by decantation and the functionalized clusters were washed by sonication and centrifugation (10 steps) in methanol, Millipore water/methanol mixture and Millipore water followed by precipitation with diluted hydrochloric acid $(0.2 \mathrm{M})$. The acidic media allowed the protonation of the carboxylate groups of the ligand on the nanoparticles surface, fact proven by the FT-IR measurements. After purification the gold clusters were filtered on precut membranes with pore diameters of $0.2 \mu \mathrm{m}$ and let to dry on the filters for about 24 hours within a desiccator. The resulted Au-MUA NP films were further characterized as explained in the manuscript. The entire experimental procedure was repeated at least three times and similar results were obtained for all functionalization procedures.

\section{Transmission electron microscopy.}

The samples for transmission electron microscopy (TEM) were prepared by deposition and evaporation of a drop of an aqueous solution of citrate gold nanoparticles, mercaptosuccinic functionalized gold nanoparticles, 1,4benezendimethanethiol functionalized gold nanoparticles or mercaptoundecanoic acid functionalized gold nanoparticles onto Formvar copper-carbon grids. TEM images were recorded with a Tecnai G2 microscope operating at $120 \mathrm{kV}$ with a point resolution of $0.5 \mathrm{~nm}$. The diameters of the observed metallic clusters were estimated using free software Image J.

\section{Digital images.}

Digital photographs of the gold nanoparticles solutions, powder of 1,4 benzenedimethanethiol functionalized gold clusters and Au-MUA NP films were recorded with a Canon Power Shot A550 digital camera of 7.1 megapixels.

UV-Vis spectroscopy. UV-Vis analyses were performed on a Hitachi U-3000 spectrophotometer for liquid samples and on a UV-3101PC Shimadzu spectrophotometer for solid films without dilution, using $\mathrm{BeSO}_{4}$ as reference. Spectra were collected over a 200-900 nm range for liquid samples and 300-900 $\mathrm{nm}$ range for the solid samples. The optical transparency of the films was verified by measuring the UV-Vis spectra using a glass slide as reference. 


\section{FT-IR spectroscopy measurements.}

FT-IR analyses were performed on a Spectrum RX I FT/IR System (Perkin Elmer), using dry powder of citrate gold nanoparticles, dry Au-MUA NP film, citrate or mercaptoundecanoic acid (few milligrams each sample) incorporated in $\mathrm{KBr}(170 \mathrm{mg})$ pellets.

\section{Scanning Electron Microscopy.}

High resolution (Schottky) Environmental Scanning Electron Microscope (FEI Quanta 400FEG / EDAX Genesis X4M) was used for the characterization of the Au-MUA NP films and for the determination of their thicknesses.

\section{Atomic Force Microscopy.}

AFM measurements were performed on a Molecular Imaging, PicoLe AFM. Au-MUA NP films of different thicknesses were cut into $1 \mathrm{~cm} / 1 \mathrm{~cm}$ pieces, fixed on the sample plate of the microscope and imaged. The surface topography was measured in tapping mode (in air) using silicon cantilever/tip (Nanosensors) with a resonance frequency between $115-475 \mathrm{kHz}$. The images were scanned in topography, amplitude, and phase mode with a resolution of $512 \times 512$ pixels. The AFM measurements were carried out on multiple areas of each film and the reproducibility of the measurements was confirmed.

\section{Results and discussion:}

Characterization of the trisodium citrate gold nanoparticles, the functionalized gold nanoparticles in solution and as films:

\section{Transmission Electron Microscopy measurements}

Figure 1 shows the TEM images of trisodium citrate gold nanoparticles. The sample showed monodisperse clusters with average diameters of $13.5 \mathrm{~nm}$ for a standard deviation of 1.56 (polydispersity: $11.58 \%$ ).
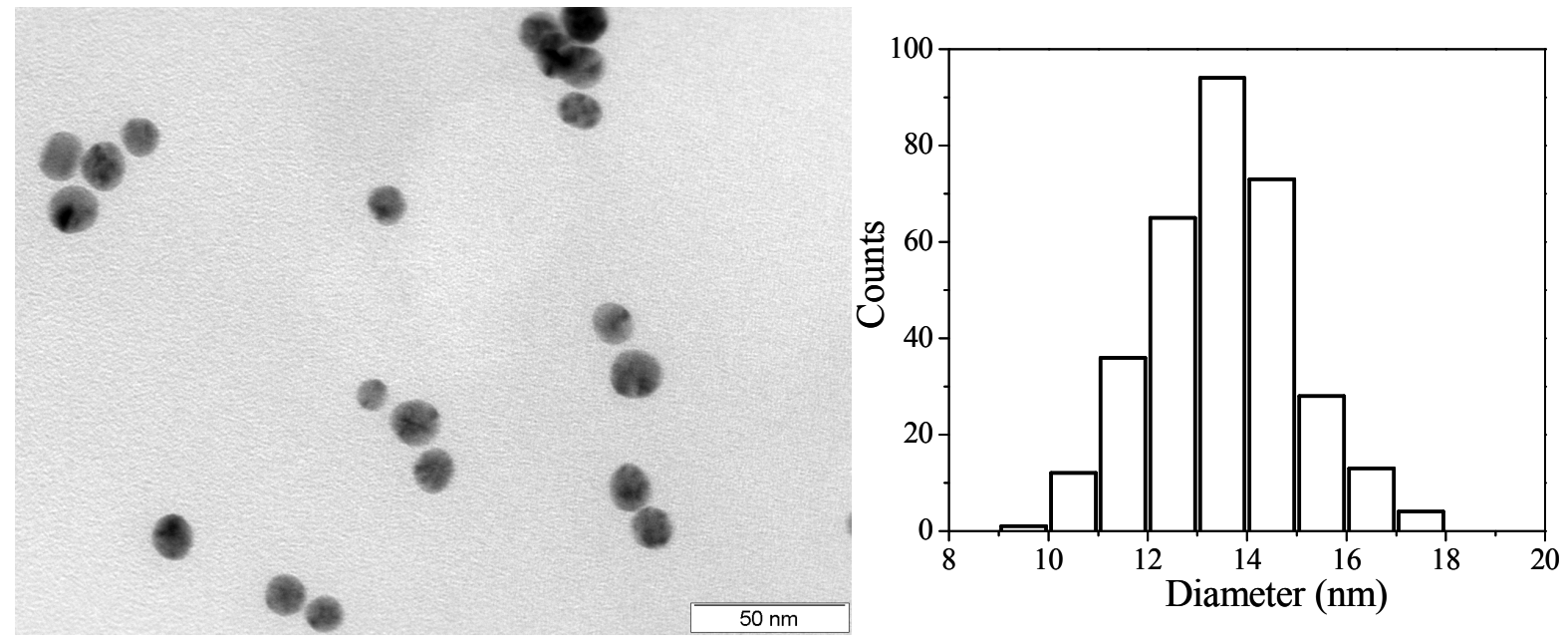

Figure 1.TEM image of trisodium citrate stabilized gold nanoparticles. Average diameters were $13.5 \mathrm{~nm}$. These nanoparticles were used for further functionalization with MSA, MUA or $1,4 \mathrm{BDMT}$.

The average diameter of the nanoparticles was determined by counting 326 nanoparticles over a range of sizes between 9.5 to $17.5 \mathrm{~nm}$.

The mercaptosuccinic acid, 1,4 Benzenedimethanethiol and mercaptoundecanoic acid functionalized gold clusters showed similar size distributions due to the fact that the starting material were the trisodium citrate stabilized gold nanoparticles.

$\boldsymbol{U V}$-Vis measurements. The place exchange reaction was monitored by UV-Vis measurements as represented in Figure 2, 3, and 4. The initial trisodium citrate stabilized gold nanoparticles showed 
a maximum absorbance peak at $520 \mathrm{~nm}$ (solution of red wine color in Figure 2a, 3a, 4a).The MSA functionalized gold nanoparticles showed a peak at $520 \mathrm{~nm}$ together with a shift of the maximum absorbance at $625 \mathrm{~nm}$ (spectra and solution of blue color in Figure 2b). The change of ligand determined a change of the optical properties of the clusters, as it could be expected, but contrarily of the well known brownish color of small (1-3 nm) mercaptosuccinic acid stabilized gold nanoparticles,[29] larger sizes of the gold clusters determined a change in color of the final solution to blue which is unexpected. After purification and drying, the MSA functionalized gold nanoparticles were analyzed by transmission electron microscopy. As it can be observed in Figure 2, right image, the MSA functionalized gold clusters formed aggregates upon drying due to the $\mathrm{H}$ bonding established between the carboxylic groups of the MSA on their surface, but no consistent films.
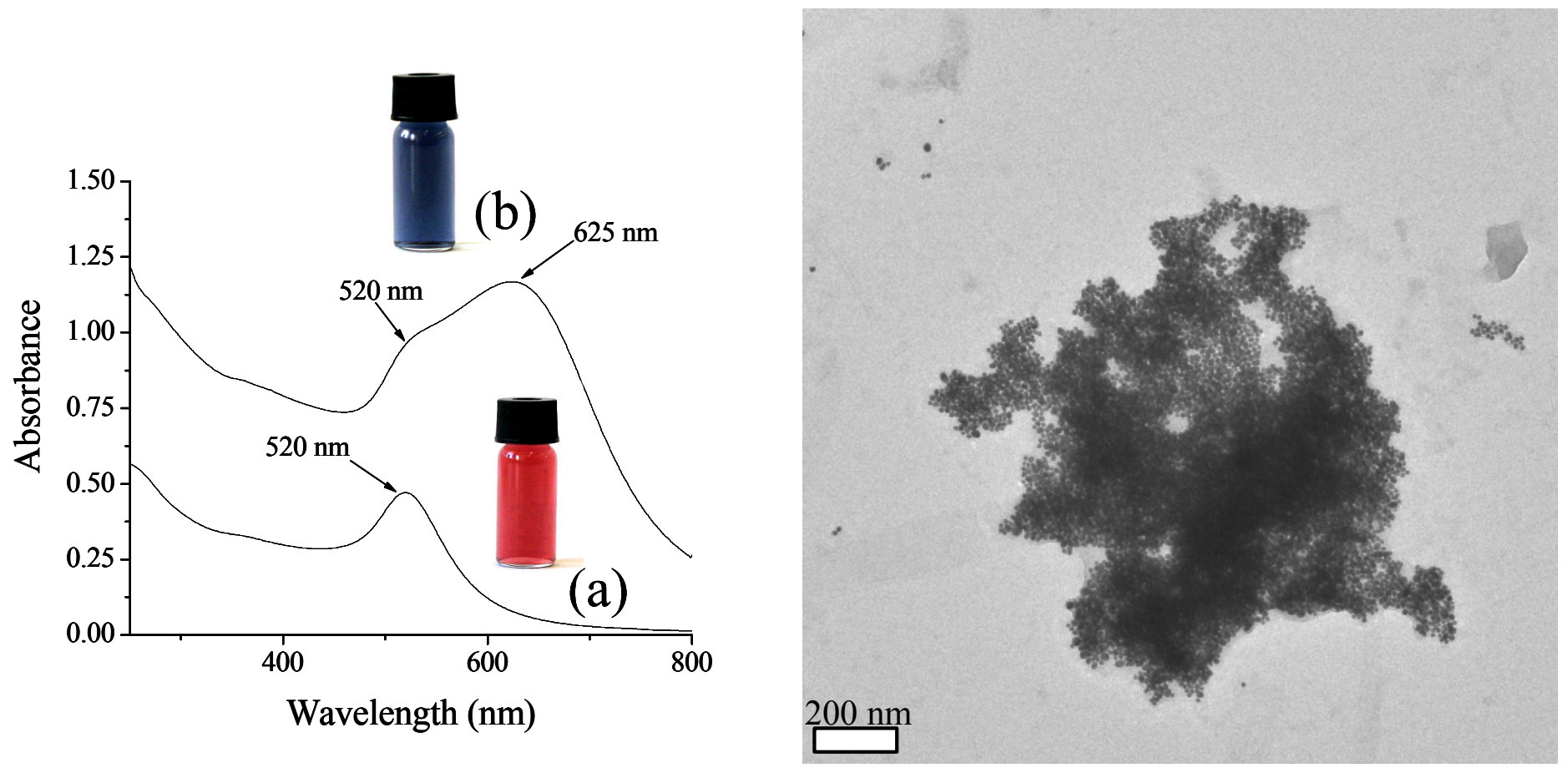

Figure 2. UV-Vis spectra (left) of trisodium citrate gold nanoparticles (a) and mercaptosuccinic acid functionalized gold nanoparticles (b). Right: TEM image of purified MSA functionalized gold nanoparticles dissolved in water.

The 1,4 Benzenedimethanethiol used for the place exchange reaction, determined the change of solution color from red to dark purple together with a shift of the maximum absorbance peak from $520 \mathrm{~nm}$ (trisodium citrate gold nanoparticles, Figure 3a) to $536 \mathrm{~nm}(1,4$ BDMT functionalized gold nanoparticles solution, Figure $3 b$ ). The gold clusters were purified by sonication and centrifugation in methanol, and filtered on precut membranes. The drying of the resulted clusters yielded powders of purple color, but no film formation. Although the ligand is a dithiol and a covalent binding could be expected between neighbor nanoparticles, TEM images (Figure 3, right image) showed only binding between several particles but no networks formation which could lead to further film growth. 


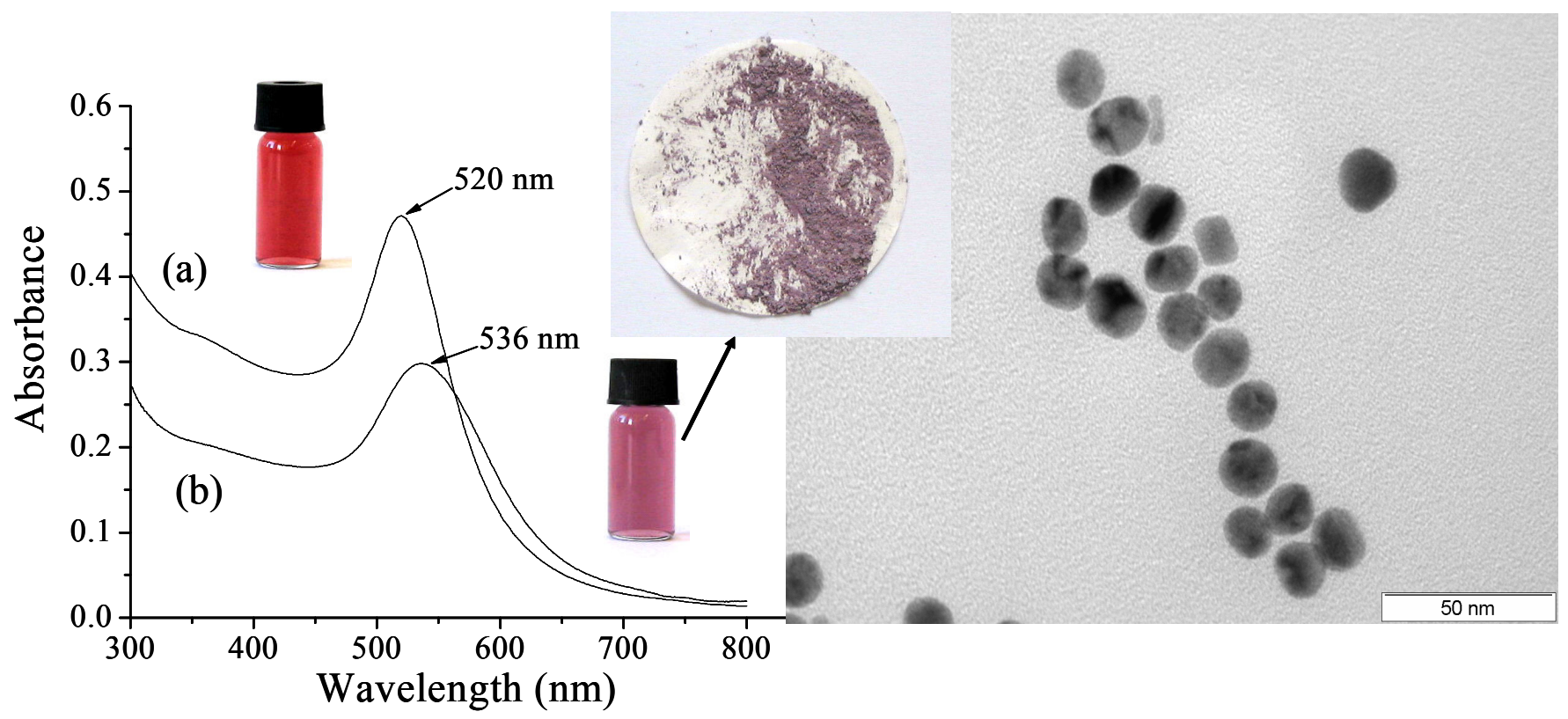

Figure 3. Left: UV-Vis spectra of trisodium citrate stabilized gold nanoparticles (a) and 1,4benezenedimethanethiol functionalized gold nanoparticles (b).Inset in the middle: powder of 1,4 BDMT functionalized gold nanoparticles and the right image represents the TEM image of same nanoparticles dissolved in methanol.

The third ligand used for the place exchange reaction, the mercaptoundecanoic acid, determined a weak shift of the maximum absorbance peak from $520 \mathrm{~nm}$ (solution of red color in Figure 4a) to $523 \mathrm{~nm}$, (solution of pink purple color in Figure 4b). After purification and drying, the MUA functionalized gold nanoparticles were filtered on pre-cut membranes and dried. Interestingly, upon drying these gold clusters formed solid films which showed a greater shift of the maximum absorbance peak either at $556 \mathrm{~nm}$ for a thick film (Figure 5a, weight film $=24.7 \mathrm{mg}$ ) or at $537 \mathrm{~nm}$ for a thin film (Figure $5 \mathrm{~b}$, weight film $=3.4 \mathrm{mg}$ ) due to the reduced particle-particle distance within the films. As proven by the optical spectra, the replacement of the citrate by mercaptoundecanoic acid, during the place exchange reaction, was successfully accomplished. TEM image (Figure 4, right) showed slight aggregations, due to the low concentration of the MUA functionalized gold clusters on the grids. The Au-MUA NP films obtained were further characterized by FT-IR spectroscopy, Scanning electron microscopy and Atomic Force Microscopy.

\section{FT-IR spectroscopy measurements:}

Figure 6 represents the FT-IR spectra of trisodium citrate (a), citrate stabilized gold nanoparticles, (b) mercaptoundecanoic acid (c), and Au-MUA NP film (d). The molecular structure of the sodium citrate was confirmed by the following frequencies of vibrations: a medium stretching vibration of water molecule at 3268-3425 $\mathrm{cm}^{-1}$ (between $3200-3600 \mathrm{~cm}^{-1}$ ), a medium stretching vibrations of the aliphatic $v_{\mathrm{C}-\mathrm{H}}$ bond between $2964-2924 \mathrm{~cm}^{-1}$, a strong asymmetric vibration of the $v_{\mathrm{C}=\mathrm{O}}$ group at $1590 \mathrm{~cm}^{-1}$ (between $1695-1540 \mathrm{~cm}^{-1}$ ) which proves the existence of the citrate as a salt, a strong stretching symmetric vibration of the $v_{\mathrm{C}=\mathrm{O}}$ group at $1394 \mathrm{~cm}^{-1}$ (between 1440-1335 $\mathrm{cm}^{-1}$ ) and scissor vibration of $\mathrm{COO}^{-}$group at $618 \mathrm{~cm}^{-1}$ and $844 \mathrm{~cm}^{-1}$ (between $860-615 \mathrm{~cm}^{-1}$ ). The vibration of $\mathrm{O}-\mathrm{H}$ group, $v_{\mathrm{C}-\mathrm{O}-\mathrm{H}}$, gives the in-plane and out-of-plane deformation vibration bands at $1306,1158,1282 \mathrm{~cm}^{-1}$. 


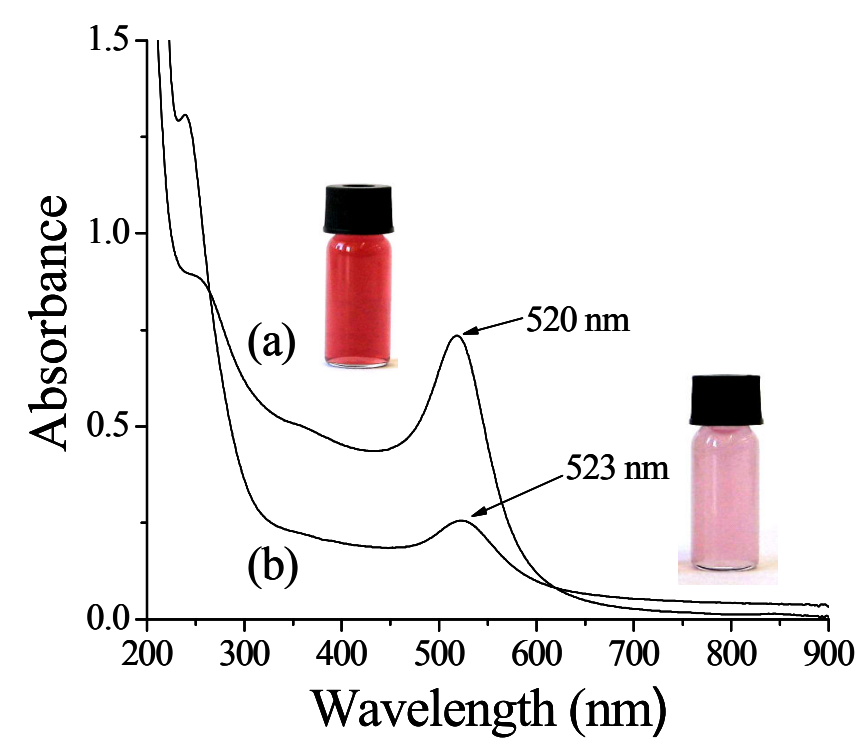

Figure 4. Right: UV-Vis spectra and digital images of citrate stabilized gold nanoparticles solution (a), mixture of citrate stabilized gold nanoparticles solution with MUA (b). Left: TEM image of MUA functionalized gold nanoparticles dissolved in diluted aqueous TEAOH.

(a)
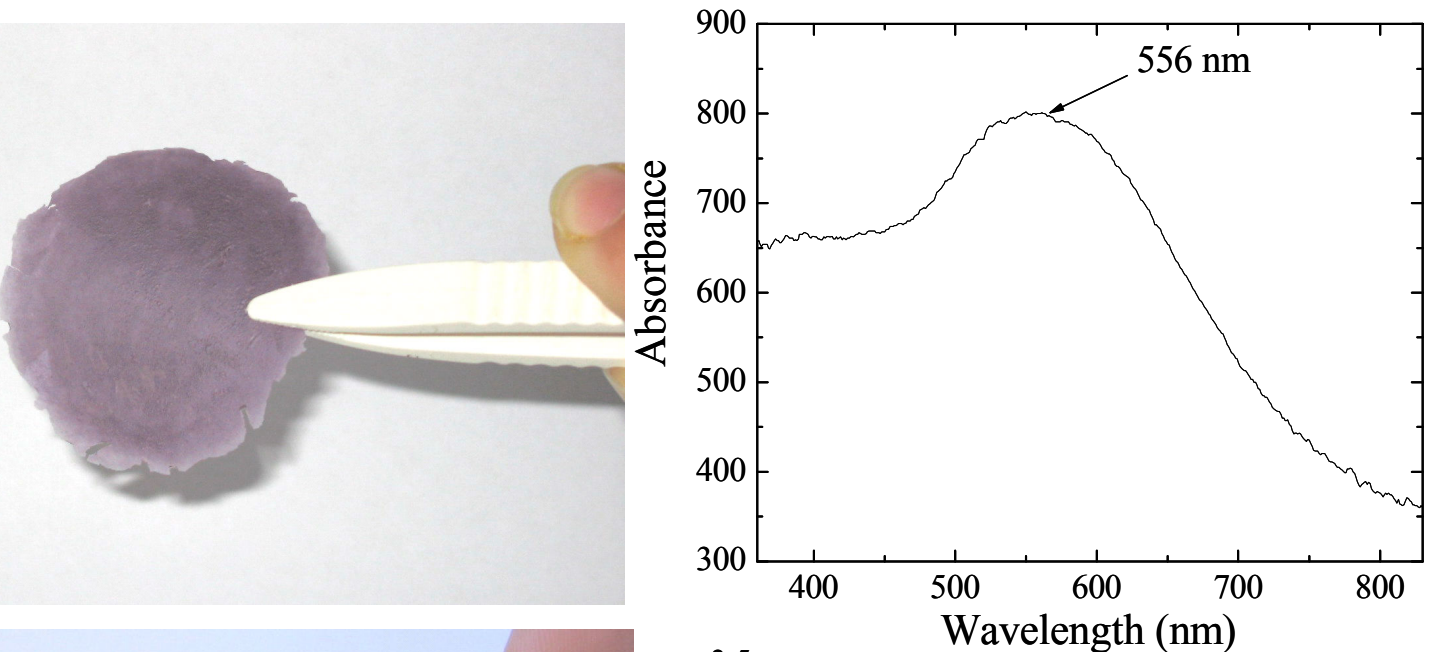

(b)

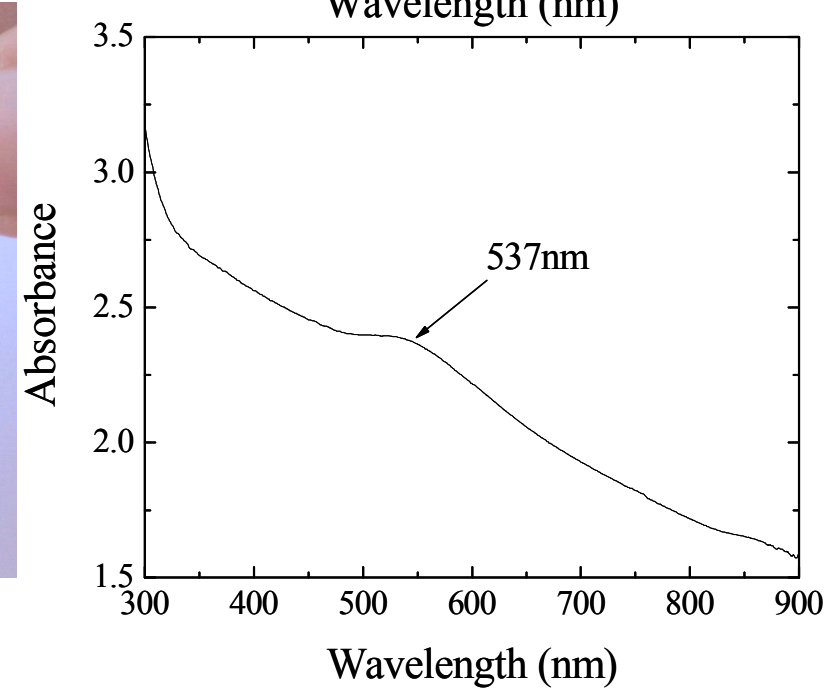

Figure5. Digital images of Au-MUA NP films of $24.7 \mathrm{mg}$ (a) and $3.4 \mathrm{mg}$ (b) and their corresponding optical spectra. 
The FT-IR spectrum of the citrate stabilized gold nanoparticles showed similar peaks, slightly diminished, as the FT-IR spectra of the sodium citrate proving the presence of the trisodium citrate on the gold clusters surface (Figure 6b). In order to verify the functionalization of the gold clusters using MUA as the incoming ligand, FT-IR spectra were recorded for the resulted Au-MUA NP film and for the MUA itself.
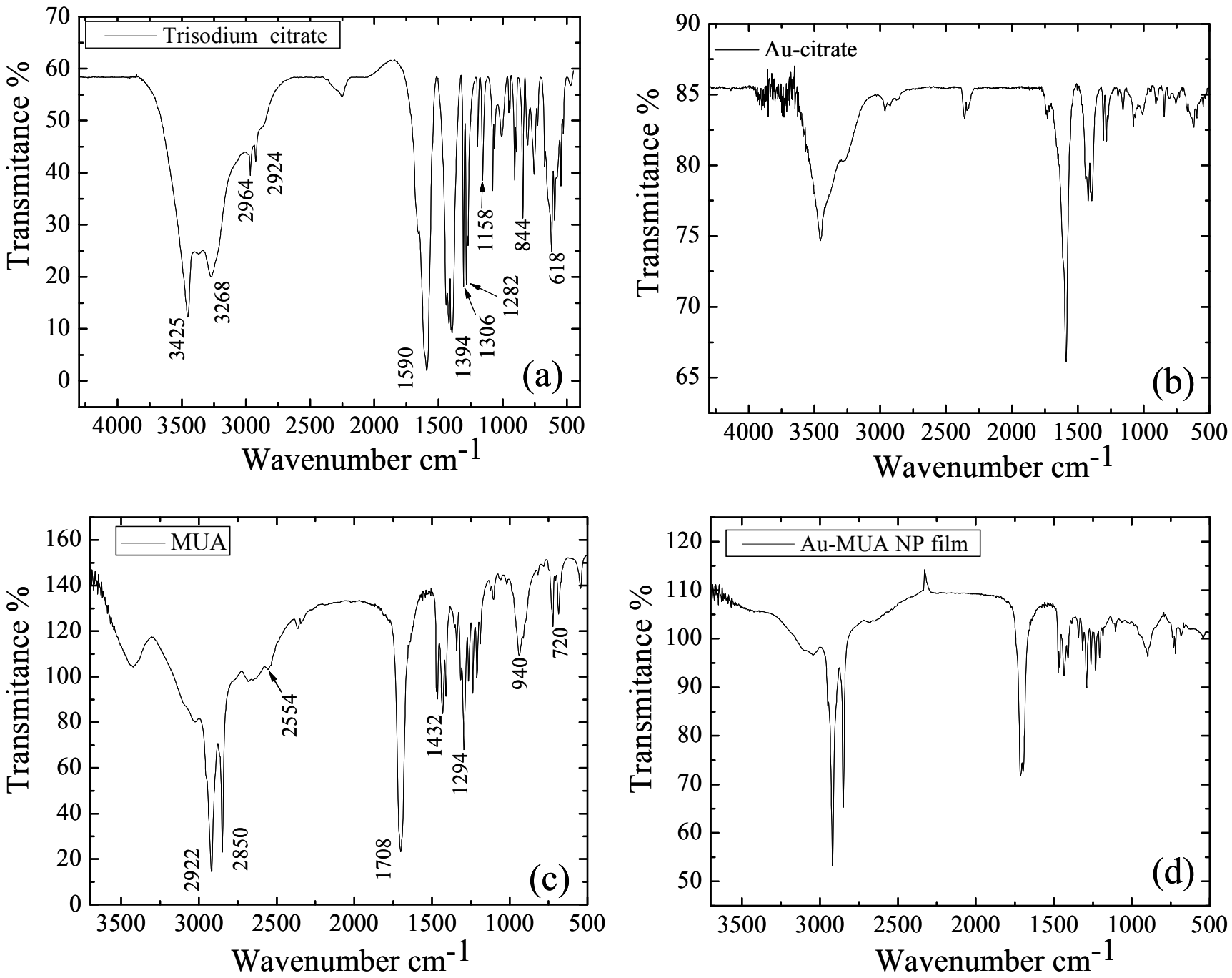

Figure 6.FT-IR spectra of sodium citrate (a), citrate stabilized gold nanoparticles, (b) mercaptoundecanoic acid (c), and Au-MUA NP film (d).

As shown in Figure $6 \mathrm{c}, \mathrm{d}$, the $v_{\mathrm{O}-\mathrm{H}}$ broad stretching band superimposed on the aliphatic $v_{\mathrm{C}-\mathrm{H}}$ stretch vibration around $3000 \mathrm{~cm}^{-1}$ confirms the presence of a long aliphatic chain terminated by $\mathrm{COOH}$ group and the removal of the short aliphatic chain of the trisodium citrate. Compared to the pure MUA spectrum (Figure 6c), the similar vibrations are observed in the Au-MUA NP film spectrum: $v_{\mathrm{C}-\mathrm{H}}$ saturated vibration stretching at $2800-3100 \mathrm{~cm}^{-1}$; the $v_{\mathrm{S}-\mathrm{H}}$ stretching vibration at $2554 \mathrm{~cm}^{-1}$ for the pure MUA disappears when the thiol is adsorbed on the Au-MUA NPs surface. This represents strong evidence that the thiol is bounded on the gold nanoparticles' surface trough the sulfur atom group. The $v_{\mathrm{C}=\mathrm{O}}$ stretching vibration appears as a strong peak at $1708 \mathrm{~cm}^{-1}$ in the MUA and Au-MUA NP film spectra and clearly indicates that the pure MUA molecules and the MUA molecules on the Au NPs surface within the Au MUA NP film exist as dimmers. This proves 
that the ligands on the Au MUA NPs surface exist mainly in the form of carboxylic acid and explains the films formation through hydrogen bonding. The fingerprint region of the FT-IR spectra shows bands at $1432 \mathrm{~cm}^{-1}$ and $1294 \mathrm{~cm}^{-1}$ which correspond to the interacting $v_{\mathrm{C}-\mathrm{O}}$ stretch and inplane $v_{\mathrm{C}-\mathrm{O}-\mathrm{H}}$ deformation vibration. The peak at $940 \mathrm{~cm}^{-1}$ is characteristic of out-of-plane $v_{\mathrm{O}-\mathrm{H}}$ bending mode in intermolecular hydrogen bonding dimmer structure. These peaks are slightly diminished in the spectrum of Au-MUA NP film and confirm the presence of the thiol as carboxylic acid on the gold nanoparticles surfaces. These measurements confirmed also an efficient replacement of the trisodium citrate (the outgoing ligand) by the mercaptoundecanoic acid (the incoming ligand) during the place exchange reaction and the presence of the carboxylic groups generating the Au-MUA NP films.

\section{Scanning Electron Microscopy and Atomic Force Microscopy measurements:}

In order to obtain information about the film morphology, structure, topography and thickness, SEM (Figure 7) and AFM measurements (Figure 8) were performed. Figure 7 represents the SEM images of the Au-MUA NP film (weight=24.7 mg) illustrated in Figure 5a which proved to have a layered structure and a thickness varying between 10 to $20 \mu \mathrm{m}$.
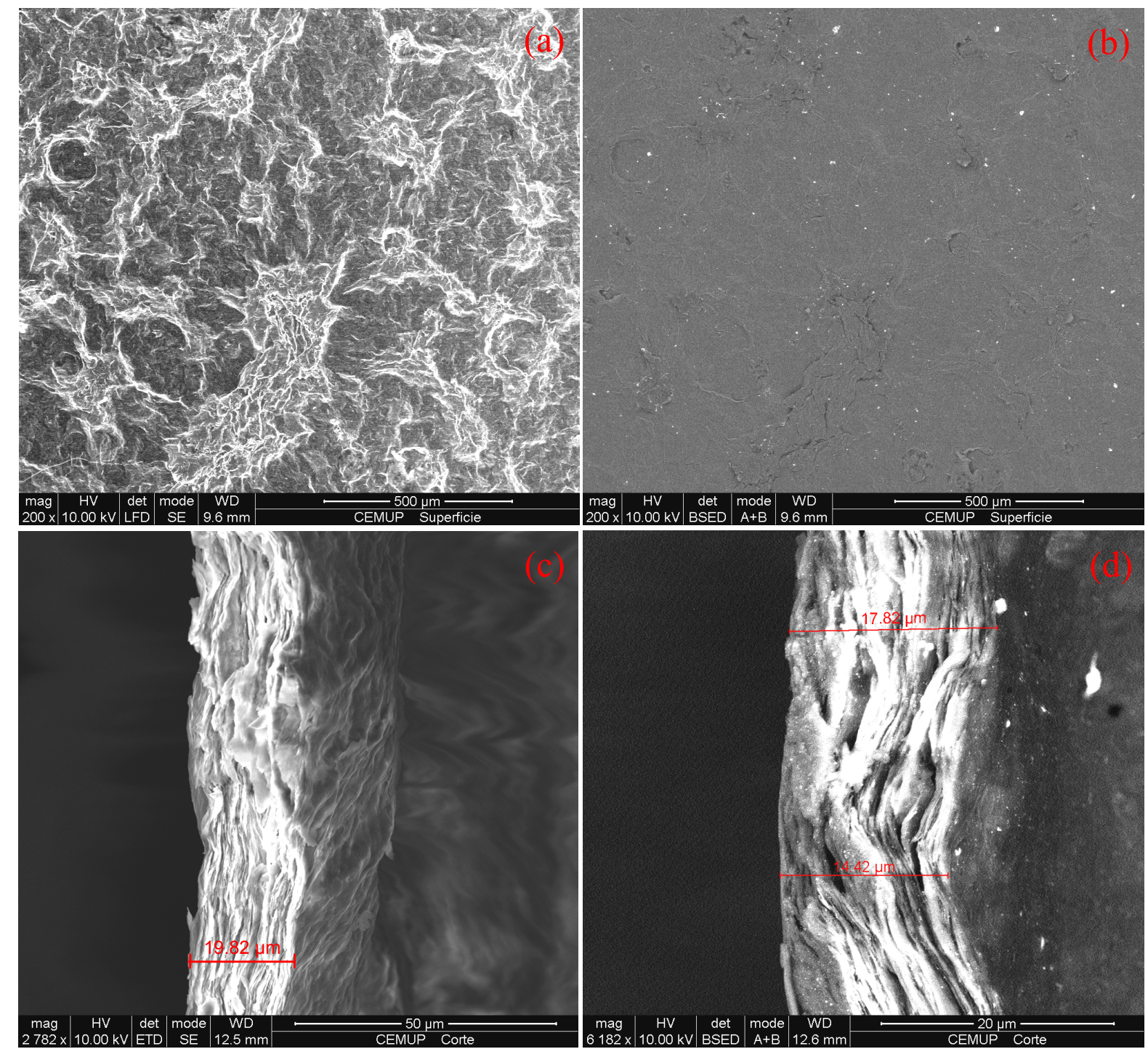

Figure 7. SEM images representing the features of the thick Au-MUA NP film surface $(a, b)$, the layered structure of the film and its thickness (c, d). The scale bars were $500 \mu \mathrm{m}$ for the images (a), (b), $50 \mu \mathrm{m}$ for (c) and $20 \mu \mathrm{m}$ for (d), respectively. The thickness of the film varied between 10 to $20 \mu \mathrm{m}$. 
The SEM backscattered electron diffraction images (Figure $7 \mathrm{~b}, \mathrm{~d}$ ) showed agglomerations of the metallic clusters both on the film surface as well as within the film structure which is consistent with hydrogen bonding formation on the vertical and horizontal planes generating the new material. In order to determine the surface roughness and the topographic features of the Au-MUA NP films, tapping mode AFM measurements were performed (Figure 8).
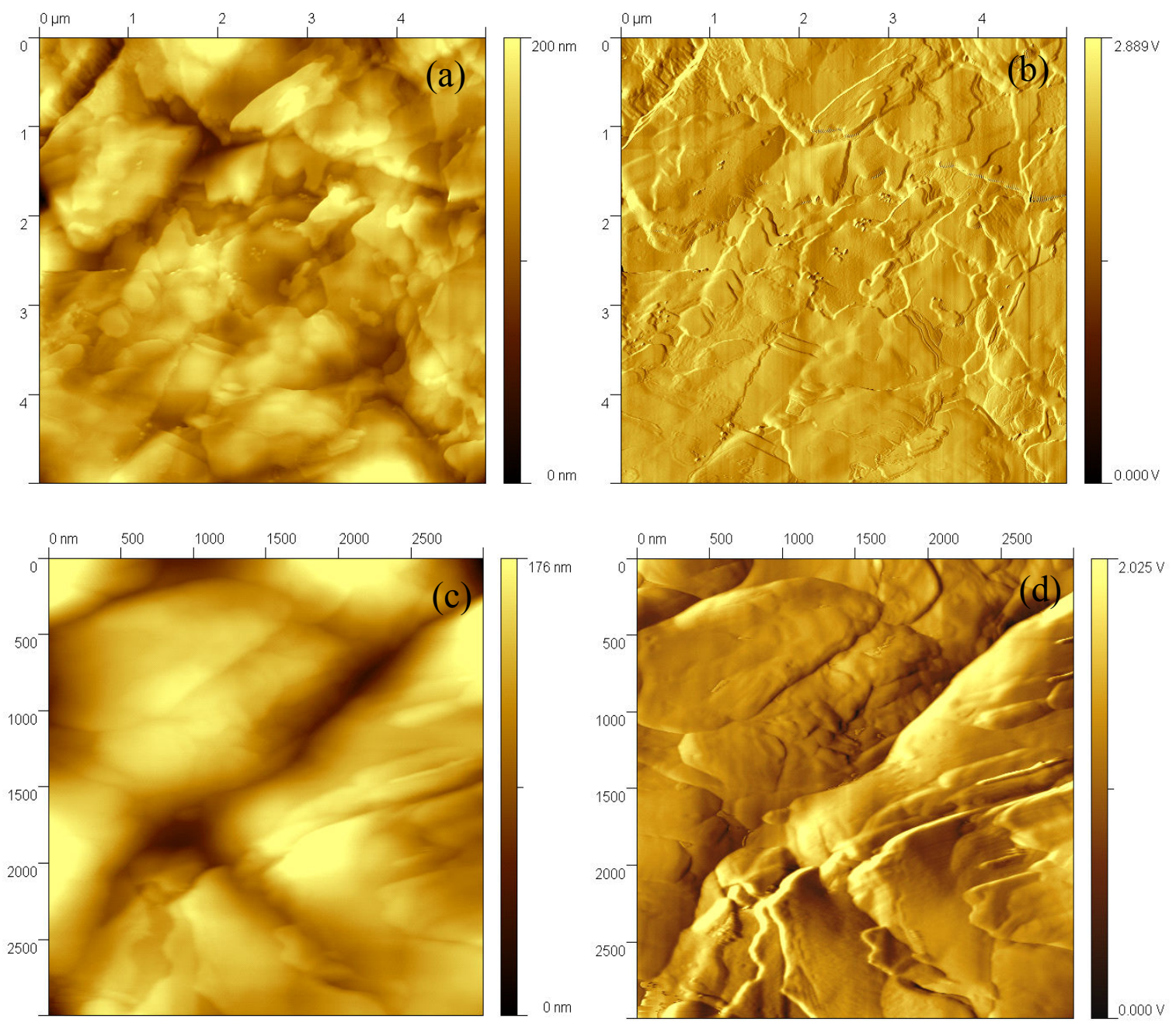

Figure 8. Topographic tapping mode AFM images of the thick (a) and thin (b) Au-MUA NP films represented in Figure 5a, b and the corresponding amplitude images (b, d). Scan size: $5 \mu \mathrm{m}$ (a) and $3 \mu \mathrm{m}(\mathrm{b})$, respectively.

It was observed that the topography of the thick film (Figure 8a, b) consisted of soft regions with layered features and agglomeration of the clusters which confirmed the results obtained by the SEM measurements. The root-mean-squared roughness of this Au-MUA NP film was $29.34 \mathrm{~nm}$ for a scan size of $5 \mu \mathrm{m}$, which proves a very soft topography. Layered structure but no agglomerations of the gold clusters, on the film surface were observed for very thin films. The root-mean-squared roughness of the thin Au-MUA NP film represented in Figure 8c,d was $18.50 \mathrm{~nm}$ for a scan size of $3 \mu \mathrm{m}$. Interestingly, the physical properties of the Au-MUA NP films were dependent on their thicknesses. For example, the thinner film $(1-4 \mu \mathrm{m}$, ) showed a maximum absorbance peak at $537 \mathrm{~nm}$ (Figure $5 \mathrm{~b})$ compared to the thicker film $(10-20 \mu \mathrm{m})$ represented in Figure 5a, which showed a higher red-shift of the surface plasmon band (maximum absorbance at $556 \mathrm{~nm}$ ). Both films showed 
optical transparency. The red-shift of the surface plasmon band is due to the reduced nanoparticlenanoparticle distance (i.e., more dense packing) in these films as compared with that of Au-MUA NPs dispersed in aqueous TEAOH solution $0.005 \%$, v/v $(523 \mathrm{~nm})$. The variation of the optical properties with the thicknesses proved by these films is consistent with previous observations for ultrathin Au films obtained by evaporation on mica and annealing.[30] Thinner films were resistant at soft mechanical manipulation; thicker films were resistant at normal mechanical manipulation, even after being packed in aluminum foil. Moreover, the Au-MUA NP films could be cut in different shapes and redissolved in diluted aqueous tetraethyl ammonium hydroxide. In our previous method of synthesis of gold nanoparticles stabilized with mercaptoundecanoic acid, [3] the amount of thiol used had as purpose the control of the size of the clusters and it was very small compared to the amount of thiol used in the present work. Previously, the clusters stabilized by MUA appeared as powders of orange or brownish color depending on their size (1-4 nm). [3] In the present work, the clusters are larger $(13.5 \mathrm{~nm})$ and the amount of ligand on the nanoparticles surface is considerably increased through the place exchange reaction, when no size control is needed but only the entire removal of the previous ligand (the trisodium citrate) is sought. It is known that thiols with long alkyl chain form compact and well organized films on flat gold substrates.[31,32] In this case, due to the small size and spherical shape of the clusters which implies increased surface area and due to the strong affinity of the thiol group toward gold atoms, the number of the ligands on the clusters surface could be denser than the number of ligands self-assembled on a flat gold substrate. This is supported also by the fact that edges and vertexes (defect sites) on the nanoparticle surface are more reactive toward the ligand binding than the terrace sites specific to a flat gold surface.[27] It seems that a larger diameter of the gold clusters and an increased number of MUA ligands on their surface determined a change of the physical properties of the Au-MUA NPs. These gold clusters organize spontaneously as superstructures based on two cumulative effects: a) due to the denser packing of the ligands on the clusters surface, van der Waals attractive forces between the alkyl chains are easily achieved and more important b) the presence of the carboxylic groups leads to the formation of hydrogen bonds between the mercaptoundecanoic acid molecules self-assembled on the gold nanoparticles surface. Breaking a hydrogen bond requires about $5 \mathrm{kcal} / \mathrm{mol}(20 \mathrm{~kJ} / \mathrm{mol})$, compared with about $100 \mathrm{kcal} / \mathrm{mol}$ (about $400 \mathrm{~kJ} / \mathrm{mol}$ ) required to break a $\mathrm{C}-\mathrm{H}, \mathrm{N}-\mathrm{H}$, or O-H bond, but the hydrogen bonding is a strong form of intermolecular attraction having a large effect on the physical properties of organic compounds.[33] In this study, although the hydrogen bond is less strong than a covalent bond, it appears that there are enough ligands on the nanoparticles surface to achieve hydrogen bonding in the horizontal and vertical planes, leading to the formation of solid Au-MUA NP films. More detailed characterization of these Au-MUA NP films, by elemental analysis and thermogravimetric measurements, are envisaged for a more complete understanding of the ligand effect on supramolecular structures formation and film generation. Also further tests in order to study the electrical properties of these films are currently performed.

\section{Conclusions.}

Trisodium citrate gold nanoparticles of $13.5 \mathrm{~nm}$ were synthesized by Turkevich method and successively functionalized by place exchange reaction using three different incoming ligands: mercaptosuccinic acid, 1,4 benzenedimethanethiol and mercaptoundecanoic acid. The resulted clusters were characterized by UV-Vis spectroscopy and transmission electron microscopy. Although the three ligands determined a change of the physicochemical properties of the gold clusters, only the mercaptoundecanoic acid have generated soft solid films of Au-MUA NPs based on hydrogen bonding interaction as a bottom-up approach self-assembly method. These films were further characterized by UV-Vis spectroscopy, FT-IR spectroscopy, Scanning Electron Microscopy and Atomic Force Microscopy and a unique combination of excellent features were observed: homogeneity, robustness and remarkable optical properties including optical transparency, making them ideally suited for applications in the field of molecular optoelectronics. Their properties could be easily controlled by varying their thickness. Moreover, these new Au-MUA NP films could be used as interdigitated arrays for electrochemical studies. 


\section{References}

[1] Ozin, G.A.;Arsenault, A.C.; Nanochemistry-A Chemical Approach to Nanomaterials, The Royal Society of Chemistry, 2005.

[2]. A. N. Shipway, E. Katz, I. Willner, Nanoparticle Arrays on Surfaces for Electronic, Optical, and Sensor Applications, ChemPhysChem, 1, (2000), 18-52.

[3]. M. Chirea, C. M. Pereira, F. Silva, Catalytic Effect of Gold Nanoparticles Self-Assembled in Multilayered Polyelectrolyte Films, J. Phys. Chem. C ,111, (2007), 9255-9266

[4].M. Chirea, V. Garcia-Morales, J.A. Manzanares, C. Pereira, R. Gulaboski, F. Silva, Electrochemical Characterization of Polyelectrolyte/Gold Nanoparticle Multilayers SelfAssembled on Gold Electrodes, J. Phys. Chem. B, 109, (2005), 21808-21817.

[5].H. A. Santos, M. Chirea, V. Garcia-Morales, F. Silva, J.A. Manzanares, K. Kontturi, Electrochemical Study of Interfacial Composite Nanostructures: Polyelectrolyte/Gold Nanoparticle Multilayers Assembled on Phospholipid/Dextran Sulfate Monolayers at a Liquid-Liquid Interface, , J. Phys. Chem. B, 109, (2005), 20105-20114.

[6]. L. E. Russell, A. A. Galyean, S. M. Notte, M. C. Leopold, Stable Aqueous Nanoparticle Film Assemblies with Covalent and Charged Polymer Linking Networks, Langmuir, 23, (2007), 74667471

[7].C. Lu, H. Molhwald, A. Fery, Plasmon Resonance Tunable by Deaggregation of Gold Nanoparticles in Multilayers, J. Phys. Chem. C , 111, (2007), 10082-10087

[8]. K. V. Sarathy, P. J. Thomas, G. U. Kulkarni, C. N. R. Rao, Superlattices of Metal and MetalSemiconductor Quantum Dots Obtained by Layer-by-Layer Deposition of Nanoparticle Arrays, J. Phys. Chem. B, 103, ( 1999), 399-401.

[9]. M. D. Musick, C. D. Keating, M. H. Keefe, M. J. Natan, Stepwise Construction of Conductive Au Colloid Multilayers from Solution, Chem. Mater., 9, 1997, 1499-1501.

[10]. M. Wanunu, R. Popovitz-Biro, H. Cohen, A. Vaskevich, I. Rubinstein, Coordination-Based Gold Nanoparticle Layers, J.Am.Chem.Soc., 127, (2005), 9207-9215.

[11]. R. Baron, C-H. Huang, D.M. Bassani, A. Onopriyenko, M. Zayats, I. Willner, HydrogenBonded CdS Nanoparticle Assemblies on Electrodes for Photoelectrochemical Applications, Angew. Chem. Int. Ed., 44, (2005), 4010-4015.

[12]. J-P. Xu, Y. Weizmann, N. Krikhely, R. Baron, I. Willner, Layered Hydrogen-Bonded Nucleotide-Functionalized CdS Nanoparticles for Photoelectrochemical Applications, Small, 2, (2006), 1178-1182.

[13]. A.C.Templeton, W.P. Wuelfing, R.W.Murray, Monolayer-Protected Cluster Molecules, Acc.Chem.Res., 33, (2000), 27-36.

[14]. F. P. Zamborini, J. F. Hicks, R W. Murray, Quantized Double Layer Charging of Nanoparticle Films Assembled Using Carboxylate/( $\mathrm{Cu}^{2+}$ or $\left.\mathrm{Zn}^{2+}\right) /$ Carboxylate Bridges, J. Am. Chem. Soc., 122, (2000), 4514-4515

[15]. M. Brust, D. Bethell, C. J. Kiely, D. J. Schiffrin, Self-Assembled Gold Nanoparticle Thin Films with Non-metallic Optical and Electronic Properties, Langmuir, 14, (1998), 5425-5429

[16]. S. S. Kinge, M. Crego-Calama, D. N. Reinhoudt, Gold Nanoparticle Assemblies through Hydrogen-Bonded Supramolecular Mediators , Langmuir, 23, (2007), 8772-8777 
[17]. Y. Y. Pinto, J. D. Le, N. C. Seeman, K. Musier-Forsyth, T. A. Taton, R. A. Kiehl, SequenceEncoded Self-Assembly of Multiple-Nanocomponent Arrays by 2D DNA Scaffolding, Nano Lett., 5, (2005), 2399-2402.

[18].N.C.Seeman, DNA in a Material World, Nature, 421, (2003), 427-431.

[19]. H-C. Chou, C-H. Hsu, Y-M. Cheng, C.-C. Cheng, H-W. Liu, S.-C. Pu, P-T. Chou, Multiple Hydrogen Bonds Tuning Guest/Host Excited-State Proton Transfer Reaction: Its Application in Molecular Recognition, J.Am.Chem.Soc. , 126, (2004), 1650-1651

[20]. T. B. Norsten, E. Jeoung, R. J. Thibault, V. M. Rotello, Specific Hydrogen-Bond-Mediated Recognition and Modification of Surfaces Using Complementary Functionalized Polymers, Langmuir, 19, (2003), 7089-7093

[21]. S. C. Zimmerman, P. S. Corbin, Heteroaromatic Modules for Self-Assembly Using Multiple Hydrogen Bonds, Struct. Bonding (Berlin), 96 (2000), 63-94.

[22]. T. A. Taton, R. C. Mucic, C. A. Mirkin, R. L. Letsinger, The DNA-Mediated Formation of Supramolecular Mono- and Multilayered Nanoparticle Structures, J. Am. Chem. Soc., 122, (2000), 6305-6306

[23]. I.Willner, F. Patolsky, J. Wasserman, Photoelectrochemistry with Controlled DNA-CrossLinked CdS Nanoparticle Arrays, Angew.Chem.Int.Ed., 40, (2001), 1861-1864. [24]. K. Aslan, C. C. Luhrs, V. H. Perez-Luna, Controlled and Reversible Aggregation of Biotinylated Gold Nanoparticles with Streptavidin, J. Phys. Chem. B, 108, (2004), 15631-15639

[25]. E. Katz, I. Willner, Integrated Nanoparticle-Biomolecule Hybrid Systems: Synthesis, Properties, and Applications, Angew.Chem.Int.Ed. 43, (2004), 6042-6108.

[26]. R.Shenhar, T.B. Norsten, V.M.Rotello, Polymer-mediated Nanoparticle Assembly: Structural Control and Applications, Adv.Mater.,17, (2005), 657-669.

[27]. M. J. Hostetler, A. C. Templeton, R. W. Murray, Dynamics of Place-Exchange Reactions on Monolayer-Protected Gold Cluster Molecules, Langmuir, 15, (1999), 3782-3789

[28].J. Turkevich, P. C. Stevenson, J. Hiller, A Study of the Nucleation and Growth Processes in the Synthesis of Colloidal Gold, Discuss. Faraday. Soc, 11, (1951), 55-75.

[29]. S. Chen, K. Kimura, Synthesis and Characterization of Carboxylate-Modified Gold Nanoparticle Powders Dispersible in Water, Langmuir 15, (1999), 1075-1082

[30]. G. Kalyuzhny, A. Vaskevich, G. Ashkenasy, A. Shanzer, I. Rubinstein, UV/Vis Spectroscopy of Metalloporphyrin and Metallophthalocyanine Monolayers Self-Assembled on Ultrathin Gold Films, J. Phys. Chem. B, 104, (2000), 8238-8244

[31]. M. D. Porter, T. B. Bright, D. L. Allara, C. E. D. Chidseyi, Spontaneously Organized Molecular Assemblies. 4. Structural Characterization of n-Alkyl Thiol Monolayers on Gold by Optical Ellipsometry, Infrared Spectroscopy, and Electrochemistry, J.Am.Chem.Soc., 109, (1987), 3559-3568

[32]. H. O. Finklea," S. Avery, M. Lynch, Blocking Oriented Monolayers of Alkyl Mercaptans on Gold Electrodes, Langmuir, 3, (1987), 409-413.

[33] Wade, L.J.Jr., Organic Chemistry, $4^{\text {th }}$ edition, Prentice Hall, 1999, p.64-65. 